\title{
Comparison of long term outcome in patients with or without aortic ring abscess treated surgically for aortic valve infective endocarditis
}

\author{
N Danchin, G Retournay, O Stchepinsky, C Selton-Suty, P Voiriot, B Hoen, P Canton, \\ J-P Villemot, P Mathieu, F Cherrier
}

\begin{abstract}
Objective-To assess the long term prognostic significance of aortic valve ring abscess in patients with aortic endocarditis.

Patients-A consecutive series of 75 patients who had surgery for aortic infective endocarditis between 1981 and 1989; 35 had aortic ring abscesses (group 1) and 40 did not (group 2). Mean age did not differ between the two groups. Prosthetic valve endocarditis was present in $17 \%$ of group 1 and $5 \%$ of group 2. Pneumococcal or $\beta$ haemolytic streptococcal endocarditis was more common in patients with native valve endocarditis who had aortic ring abscesses (20\% v 5\%).

Design-Cohort analysis.

Results-In-hospital mortality $(11.4 \% v$ $7.5 \%)$ and 10 year survival (56\% $v 66 \%)$ were not significantly different between groups 1 and 2 . In patients with native valve endocarditis, 10 year survival was $62 \%$ and $66 \%$, respectively for patients with or without ring abscess, and 10 year reintervention-free survival was $38 \% v$ $58 \%(p=0.11)$. In these patients, the presence of an intercurrent illness, severe congestive heart failure before surgery, and use of valved conduits for surgical treatment were predictors of poorer long term survival. At follow up residual aortic regurgitation was documented in $72 \%$ of patients in group 1 and $26 \%$ in group 2 $(\mathrm{p}<0.01)$.

Conclusions-Aortic valve ring abscess is not an independent marker of poor long term outcome in patients with infective endocarditis. However, as residual aortic regurgitation appears frequent at follow up, specific surgical techniques should be considered in patients with paravalvar abscesses.

(Heart 1999;81:177-181)
\end{abstract}

Keywords: endocarditis; ring abscess; aortic valve; outcome study

Service de Chirurgie Cardiaque, CHU

Nancy-Brabois

J-P Villemot

P Mathieu

Correspondence to:

Dr Danchin.

email:

n.danchin@chu-nancy.fr

Accepted for publication 7 September 1998 optimum management. Though the presence of an aortic valve ring abscess is usually regarded as an indication for early surgical treatment as well as a marker of poor initial outcome, ${ }^{7}$ its prognostic significance over the long term has not been extensively studied. ${ }^{8}$ Our aim in the present study was therefore to analyse the in-hospital course and long term prognostic significance of valve ring abscess in patients who had undergone surgical treatment for aortic infective endocarditis.

\section{Methods}

POPULATION AND INCLUSION CRITERIA

We reviewed all medical records of patients admitted to our institution and treated surgically for infective endocarditis involving the aortic valve between January 1981 and February 1989. The patients were included if they were classified as pathologically or clinically definite, according to the Duke University classification, ${ }^{9}$ using a retrospective analysis of the hospital records.

We recorded the following baseline variables: age on admission, sex, worst preoperative New York Heart Association (NYHA) class, history of cerebral or peripheral embolism, presence of an atrioventricular block on preoperative ECG, previous history of aortic valve replacement, presence of an intercurrent illness (cancer, liver cirrhosis, or any life threatening disease), duration of preoperative antibiotic treatment, and causal microorganism.

All patients had at least one transthoracic cross sectional echocardiographic examination, but none underwent transoesophageal echocardiography during the index hospital stay, as the technique was not available at our institution at that time. Furthermore, only patients admitted in the later recruitment period had Doppler echocardiography. As previously defined, ${ }^{3}$ cross sectional echocardiographic criteria for the presence of an aortic ring abscess were the presence of a paravalvar cavity which appeared echo-free or was partially filled with necrotic debris.

The diagnosis of valve ring abscess was based upon surgical documentation of a paravalvar cavity, which could be either empty or filled with infectious or necrotic material, and was located on the aortic ring.

Two groups were defined. Group 1 comprised all anatomically documented cases of aortic valve ring abscess. Group 2 comprised all patients with aortic infective endocarditis without aortic ring abscess. Because prosthetic 
Table 1 Baseline characteristics of patients with and without aortic valve ring abscess

\begin{tabular}{|c|c|c|c|c|c|}
\hline & \multicolumn{2}{|l|}{ Group $1(n=35)$} & \multicolumn{2}{|l|}{ Group $2(n=40)$} & \multirow[b]{2}{*}{$p$ Value } \\
\hline & $\begin{array}{l}\text { 1a: native valve } \\
(n=29)\end{array}$ & $\begin{array}{l}\text { 1b: prosthesis } \\
(n=6)\end{array}$ & $\begin{array}{l}\text { 2a: native valve } \\
(n=38)\end{array}$ & $\begin{array}{l}\text { 2b: prosthesis } \\
(n=2)\end{array}$ & \\
\hline Mean (SD) age (years) & $43(17)$ & $48(18)$ & $49(13)$ & $48(28)$ & NS \\
\hline Sex (male/female) $(\%)$ & $86 / 14$ & $83 / 17$ & $74 / 26$ & $50 / 50$ & NS \\
\hline NYHA class IV & $16(55)$ & $4(67)$ & $15(40)$ & $1(50)$ & NS \\
\hline Pericarditis & $3(10)$ & 0 & $3(8)$ & 0 & \\
\hline AV block & $1(3)$ & 0 & 0 & 0 & NS \\
\hline Arterial emboli & $13(45)$ & $1(17)$ & $12(31)$ & $1(50)$ & NS \\
\hline Intercurrent illness & $3(10)$ & $1(17)$ & $5(13)$ & 0 & NS \\
\hline \multicolumn{6}{|l|}{ Causal organisms: } \\
\hline Staphylococci & $4(14)$ & $2(33)$ & $6(16)$ & 0 & NS \\
\hline Streptococci A, B, C, or pneumoniae & $7(24)^{\star}$ & 0 & $2(5)$ & 0 & $<0.05$ \\
\hline Enterococci & $3(10)$ & 0 & $7(18)$ & 0 & NS \\
\hline Viridans streptococci & $9(31)$ & 0 & $14(37)$ & 0 & NS \\
\hline Mean (SD) LVEDD (mm) & $64(9)$ & $62(5)$ & $65(7)$ & $69(13)$ & NS \\
\hline $\begin{array}{l}\text { Mean (SD) duration of preoperative } \\
\text { antibiotic treatment (days) }\end{array}$ & $31(19)^{\star}$ & $19(20)$ & $41(17)$ & $35(10)$ & 0.06 \\
\hline
\end{tabular}

Values are n (\%) unless otherwise stated.

${ }^{\star} \mathrm{p}$ value group $1 \mathrm{a} v$ group $2 \mathrm{a}$.

AV, atrioventricular; LVEDD, left ventricular end diastolic diameter; NYHA, New York Heart Association.

valve endocarditis is a recognised factor of poorer prognosis, each of the groups was subdivided into two subgroups, defined by the presence of endocarditis on a native valve (subgroups a) or on a prosthetic valve (subgroups b).

Patients who had repeated episodes of endocarditis and were readmitted during the study period were only counted once, except for one patient initially operated on for infective endocarditis without ring abscess who subsequently had a relapse with an aortic ring abscess, and who was included in both group 1 and 2 .

During the same time period, 35 patients admitted to our institution were treated medically for aortic valve endocarditis. None of these had echocardiographic evidence of aortic valve ring abscess.

FOLLOW UP

Follow up information was obtained by telephone interviews with the patients' general physicians and cardiologists. In addition, a written questionnaire was sent to all patients presumed alive. When no information could be obtained in this way, telephone interviews of the patients' families were made. Only two patients were lost to follow up, one and two years after the initial episode. These patients were included in the analyses and censored at the time of the last follow up information. The presence of residual aortic insufficiency was assessed from the last echo Doppler examination performed by the treating cardiologists.

STATISTICAL ANALYSIS

Discrete variables were compared using the $\chi^{2}$ test or, when appropriate depending on the sample sizes, Fisher's exact test. Continuous variables are expressed as mean (SD) and were compared using Student's unpaired $t$ tests. Probabilities of survival were estimated using the Kaplan-Meier method and compared using log rank tests. The univariate Cox proportional hazard model was used to determine long term prognostic indicators in the population of patients with native valve endocarditis. All $\mathrm{p}$ values less than 0.05 were considered significant.

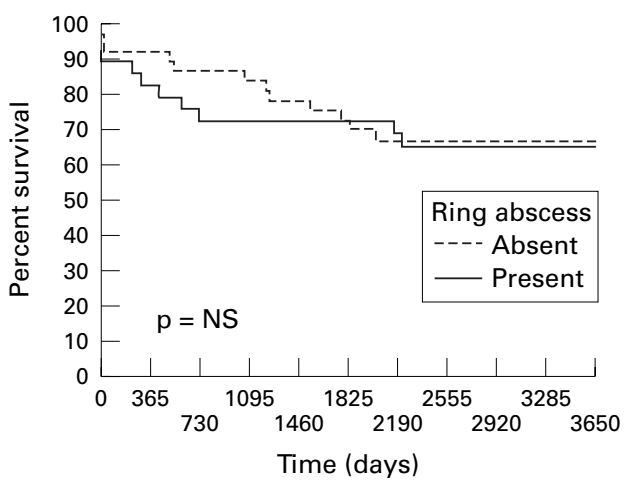

Figure 1 Long term probability of survival (Kaplan-Meier) after surgery for native aortic valve infective endocarditis, according to the presence or absence of an aortic valve ring abscess.

\section{Results}

BASELINE CHARACTERISTICS AND IN-HOSPITAL OUTCOME

The baseline characteristics of the patients are given in table 1 . There were no significant differences between groups 1 and 2, except for a higher proportion of patients with $\beta$ haemolytic streptococcal or pneumococcal infection in group 1a compared with group 2a. The time from diagnosis of infective endocarditis to aortic valve replacement was shorter in group 1a than in group $2 \mathrm{a}$.

In group 1 , one patient had aortic valve repair associated with exclusion of the abscess cavity, eight $(23 \%)$ had aortic valve replacement using a bioprosthesis, $21(60 \%)$ had aortic valve replacement with a mechanical valve, and five (14\%) required replacement of the ascending aorta with a valved conduit. In group 2 , nine patients $(22.5 \%)$ had implantation of a bioprosthesis and $31(77.5 \%)$ had a mechanical prosthesis. Four patients in each group had associated mitral valve replacement. Thirty day mortality was $11.4 \%$ (four patients) in group 1, compared with $7.5 \%$ (three patients) in group 2 (NS). The respective figures for patients with native valve endocarditis were $10.3 \%$ (three patients) and $7.9 \%$ (three patients) for groups $1 \mathrm{a}$ and $2 \mathrm{a}$ (NS). 


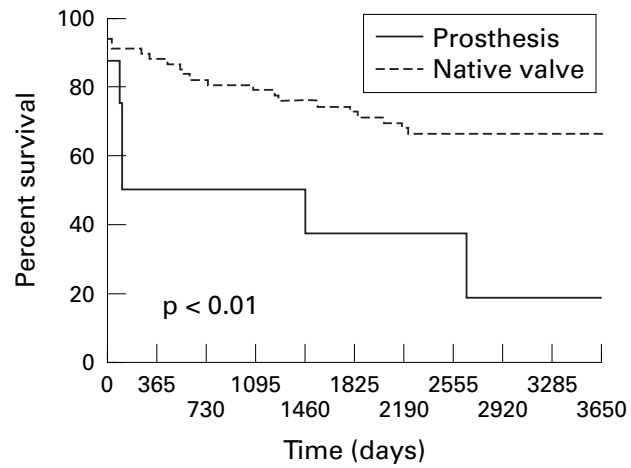

Figure 2 Long term probability of survival (Kaplan-Meier) in patients with prosthetic valve endocarditis compared with native valve endocarditis, irrespective of the presence of aortic ring abscess.

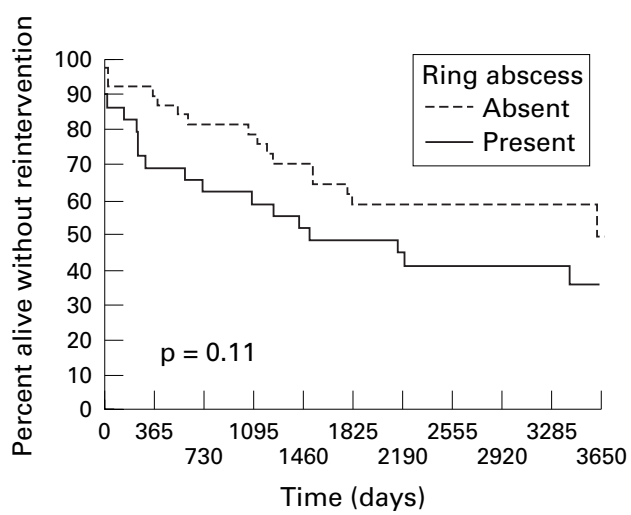

Figure 3 Long term probability of survival without reintervention after surgery for native aortic infective endocarditis, according to the presence or absence of an aortic valve ring abscess.

FOLLOW UP DATA

During the follow up period 23 patients died: 12 group 1 patients (group 1a, eight patients; group $1 \mathrm{~b}$, four patients) and 11 group 2 patients (group 2a, 10 patients; group $2 \mathrm{~b}$, one patient). Among these, the cause of death was non-cardiac in one group 1 and three group 2 patients, and two patients in each group died of

Table 2 Predictors of long term mortality in patients with native valve endocarditis

\begin{tabular}{llll}
\hline Variable (univariate analysis) & Relative risk & $95 \%$ CI & p Value \\
\hline Age (continuous) & 1.01 & 0.98 to 1.04 & NS \\
Sex (female) & 0.15 & 0.02 to 1.11 & 0.06 \\
Intercurrent illness & 2.81 & 1.03 to 7.64 & 0.04 \\
NYHA class IV & 2.45 & 1.03 to 5.84 & 0.04 \\
LV end diastolic diameter & 1.00 & 0.95 to 1.05 & NS \\
Implantation of valved conduit & 7.17 & 1.58 to 32.6 & 0.01 \\
Viridans streptococcal endocarditis & 0.45 & 0.17 to 1.23 & NS \\
Staphylococcal endocarditis & 2.08 & 0.77 to 5.65 & NS \\
Aortic ring abscess & 1.25 & 0.55 to 2.84 & NS
\end{tabular}

$\mathrm{Cl}$, confidence interval; LV, left ventricular; NYHA, New York Heart Association.

Table 3 Predictors of long term mortality or reintervention in patients with native valve endocarditis

\begin{tabular}{llll}
\hline Variable (univariate analysis) & Relative risk & $95 \% \mathrm{Cl}$ & p Value \\
\hline Age (continuous) & 1.00 & 0.97 to 1.02 & $\mathrm{NS}$ \\
Sex (female) & 0.39 & 0.14 to 1.10 & 0.07 \\
Intercurrent illness & 1.60 & 0.61 to 4.15 & $\mathrm{NS}$ \\
NYHA class IV & 1.92 & 0.99 to 3.75 & 0.05 \\
LV end diastolic diameter & 0.98 & 0.94 to 1.02 & $\mathrm{NS}$ \\
Implantation of valved conduit & 4.84 & 1.11 to 21.18 & 0.04 \\
Viridans streptococcal endocarditis & 0.61 & 0.28 to 1.30 & $\mathrm{NS}$ \\
Staphylococcal endocarditis & 2.49 & 1.07 to 5.77 & 0.03 \\
Aortic ring abscess & 1.61 & 0.83 to 3.10 & NS \\
\hline
\end{tabular}

$\mathrm{Cl}$, confidence interval; LV, left ventricular; NYHA, New York Heart Association. haemorrhagic complications while they were on oral anticoagulant treatment. Eight group 1 patients, all belonging to group $1 \mathrm{a}$, had repeat surgery on the aortic valve, compared with three in group 2, all belonging to group $2 \mathrm{a}$ $(\mathrm{p}=0.06)$. Similarly, eight group 1 patients had recurrent endocarditis, compared with five in group 2 (NS).

The clinical status at follow up was obtained in 20 group 1 patients and 24 group 2 patients. In addition, echocardiographic data were available in 18 group 1 and 23 group 2 patients. NYHA dyspnoea class III or IV was present in five group 1 and nine group 2 patients (NS). Residual aortic regurgitation ( $>$ grade 1 ) was documented on Doppler echocardiography in 13 group 1 patients and six group 2 patients $(\mathrm{p}<0.01)$.

Ten year probability of survival was not significantly different in groups $1(56 \%)$ and 2 $(66 \%)$. In patients with native valve endocarditis, 10 year probability of survival was $62 \%$ for patients with an aortic ring abscess (group 1a) and $66 \%$ for those without ring abscess (group 2a) (fig 1). In contrast, 10 year probability of survival was significantly lower in patients with prosthetic endocarditis (25\%), compared with those with native valve endocarditis (64\%) ( $p=0.0054$ ) (fig 2).

Reintervention-free survival was lower in group $1(29 \%)$ than in group $2(48 \%)$ $(\mathrm{p}<0.05)$, and survival without relapse or reintervention also tended to be poorer in group $1(29 \% v 46 \%, \mathrm{p}=0.06)$. A similar trend was observed in patients with native valve endocarditis, though the difference for reintervention-free survival was no longer significant $(38 \%$ v $58 \%, p=0.11$ ) (fig 3 ).

PREDICTORS OF SURVIVAL AND REINTERVENTION-FREE SURVIVAL IN PATIENTS WITH NATIVE VALVE ENDOCARDITIS

By univariate analysis, presence of an intercurrent illness and congestive heart failure (NYHA class IV) during the index hospital stay, as well as the type of prosthesis used (valved conduit $v$ mechanical prosthesis or bioprosthesis) were all associated with a poorer long term survival. Female sex was of borderline significance $(\mathrm{p}=0.06)$ (table 2$)$.

By univariate analysis, reintervention-free survival was related to several baseline variables, including staphylococcal endocarditis, NYHA class IV, and type of prosthesis used (table 3).

\section{Discussion}

Aortic valve ring abscess is considered to be a major complication of aortic infective endocarditis. The initial clinical presentation is usually severe, with the rapid onset of congestive heart failure but the clinical features do not usually allow an accurate diagnosis to be made. ${ }^{10}$ Its early recognition, however, has been made possible by the development echocardiographic and particularly transoesophageal echographic techniques. $^{3-6}$ The causal organisms are not very different from those of aortic valve endocarditis without ring abscess; pneumococcal endocarditis, however, is often complicated by the devel- 
opment of ring abscesses, ${ }^{11}$ and it appears from our findings that the same holds true for other $\beta$ haemolytic streptococci, which, in spite of their high sensitivity to penicillin, can provoke rapid and extensive destruction of paravalvar tissue. The presence of a paravalvar abscess is usually considered an indication for early surgical treatment ${ }^{7-8}$; in the series from Aguado et $a l^{4}{ }^{4}$ nine of 10 patients with a paravalvar abscess who were not operated on died in hospital. However, in a recent French multicentre survey, ${ }^{12}$ the in-hospital mortality was similar in patients treated surgically (23\%) and medically $(18 \%)$. Though the operative mortality is high, ranging from $11 \%$ to $30 \%,{ }^{13-15}$ in our series it was not significantly different from aortic endocarditis without ring abscess.

Several previous studies have analysed the long term outcome of aortic valve endocarditis. ${ }^{16-19}$ In our study, however, we specifically analysed the long term outcome according to the presence or absence of an aortic annular abscess. We show that, contrary to what might have been expected, the presence of an aortic ring abscess does not have independent adverse prognostic significance in terms of overall survival. In a recent study of late survival after surgical treatment of culture positive endocarditis involving either the mitral or aortic valve, Mullany et al also found that the presence of a ring abscess was not a determinant of poor long term outcome by multivariate analysis, though it appeared related to late mortality by univariate analyses. ${ }^{20}$ Prosthetic valve endocarditis and congestive heart failure (NYHA class IV), in contrast, have a deleterious influence in terms of both overall and reintervention-free survival, independent of the presence of a ring abscess. These findings are in keeping with previous series reporting a distinctly worse prognosis in patients with prosthetic valve endocarditis ${ }^{17-21}$ or severe congestive heart failure. ${ }^{16-21}$ Interestingly, a recent report from Lytle et al on the long term outcome after reoperation for prosthetic valve endocarditis documented a satisfactory survival at 10 years $(60 \%)$, and the extension of infection beyond the prosthesis, though correlated with prognosis on univariate analyses, was not a determinant of late outcome after multivariate adjustment. ${ }^{22}$

Although the presence of a ring abscess was not an independent predictor of long term outcome in our present series, it was strongly associated with residual aortic regurgitation on follow up Doppler echocardiographic examination $(72 \%$ of patients alive, compared with $26 \%$ of the patients without aortic ring abscess). This finding - already described in a series reported by John et al, ${ }^{13}$ who observed residual aortic regurgitation in $57 \%$ of patients with an aortic ring abscess compared with $8.7 \%$ of those without ring abscess-should argue for more radical or specific surgical approaches in patients with paravalvar abscesses. Recently, Glazier et al described their results using antibiotic impregnated homograft aortic root replacement with reimplantation of the coronary arteries ${ }^{14}$; in-hospital mortality was high $(30 \%)$, but event-free survival at five years in hospital survivors was excellent (81\%). In a similar attempt to achieve radical results, d'Udekem et al described a series of 70 patients with paravalvar abscesses (59 aortic ring abscesses) who were treated with a wide resection of the abscess followed by reconstruction of the annulus with autologous or bovine pericardium and valve replacement ${ }^{19}$; eight year survival was $64 \%$ and only eight patients had recurrent infective endocarditis.

\section{CONCLUSION}

In patients operated on for aortic infective endocarditis, the presence of an aortic ring abscess does not imply a poorer long term (10 year) survival, although it is more commonly associated with the need for repeat interventions. In contrast, the high frequency of residual aortic regurgitation in these patients suggests the need for specific surgical techniques when an aortic ring abscess is present.

1 Arnett EN, Roberts WC. Valve ring abscess in active infective endocarditis. Frequency, location and clues to clinical diagnosis from the study of 95 necropsy patients. Circulation 1976;54:140-5

2 Scanlan JG, Seward JB, Tajik AJ. Valve ring abscess in infective endocarditis: visualisation with wide-angle twodimensional echocardiography. Am $\mathcal{F}$ Cardiol 1982;49: 1794-800

3 Neimann JL, Danchin N, Godenier JP, et al. Twodimensional echocardiographic recognition of aortic valve ring abscess. Eur Heart f 1984;5(suppl C):59-65.

4 Aguado JM, Gonzalez-Vilchez F, Martin-Duran R, et al. Perivalvular abscesses associated with endocarditis. Clinical features and diagnostic accuracy of twodimensional echocardiography. Chest 1993;104:88-93.

5 Daniel WG, Mügge A, Martin RP, et al. Improvement in the diagnosis of abscesses associated with endocarditis by diagnosis of abscesses associated with endocarditis by transesophage

6 Rohmann S, Erbel R, Mohr-Kahaly S, et al. Use of transoesophageal echocardiography in the diagnosis of abscess in infective endocarditis. Eur Heart f 1995;16(suppl B):54-62.

7 Michel PL, Vitoux B, Hage A, et al. Early or delayed surgery in acute native aortic valve endocarditis. In: Hortskotte $\mathrm{D}$, endocarditis. London: ICR Publishers, 1991:220-8.

8 Amrani M, Schoevaerdts JC, Rubay J, et al. Surgical treatment of acute native aortic valvular infective endocarditis: long-term follow-up. Cardiovasc Surg 1995;3: 579-81.

9 Durack DT, Lukes AS, Bright DK, et al. New criteria for Durack DT, Lukes AS, Bright DK, et al. New criteria for
diagnosis of infective endocarditis: utilization of specific diagnosis of infective endocarditis: utilization of specife

10 Blumberg EA, Karalis DA, Chandrasekaran K, et al. Endocarditis-associated paravalvular abscesses. Do clinical parameters predict the presence of abscesses? Chest 1995;107:898-903.

11 Ugolini V, Pacifico A, Smitherman TC, et al. Pneumococcal endocarditis update: analysis of 10 cases diagnosed between 1975 and 1984. Am Heart f 1986;112:813-19.

12 Lesbre JP, Tribouilloy C, Jaubourg ML, et al. Les abcès para-annulaires; à propos de 59 cas. Etude multicentrique. Arch Mal Cœur 1995;88:321-8.

13 John RM, Pugsley W, Treasure T, et al. Aortic root complications of infective endocarditis. Influence on surgical outcome. Eur Heart f 1991;12:241-8.

14 Glazier JJ, Verwilghen J, Donaldson RM, et al. Treatment of complicated prosthetic aortic valve endocarditis with complicated prosthetic aortic valve endocarditis with
annular abscess formation by homograft aortic root replacement. F Am Coll Cardiol 1991;17:1177-82.

15 Jault F, Gandjbakhch I, Chastre JC, et al. Prosthetic valve endocarditis with ring abscesses. Surgical management and long-term results. F Thorac Cardiovasc Surg 1993;105: 1106-13.

16 Kimose HH, Lund O, Kromann-Hansen O. Risk factors for early and late outcome after surgical treatment of native valve endocarditis. Scand $f$ Thorac Cardiovasc Surg 1990;24:11-20.

17 Aranki SF, Santini F, Adams DH, et al. Aortic valve endocarditis. Determinants of early survival and late morbidity. Circulation 1994;90(suppl II): II-175-82.

18 Delahaye F, Ecochard R, De Gevigney G, et al. The long term prognosis of infective endocarditis. Eur Heart $\mathcal{F} 1995$; 16(suppl B): $48-53$.

19 d'Udekem Y, David TE, Feindel CM, et al. Long-term results of operation for paravalvular abscesses. Ann Thorac Surg 1996;62:48-53. 
20 Mullany CJ, Chua YL, Schaff HV, et al. Early and late survival after surgical treatment of culture-positive active Tho Clin Proc 1995:70:517-25.

21 Otaki M. Prosthetic valve endocarditis: surgical procedures and clinical outcome. Cardiovasc Surg 1994;2:212-15.

22 Lytle BW, Priest BP, Taylor PC, et al. Surgical treatment of prosthetic valve endocarditis. If Thorac Cardiovasc Surg 996;111:198-207.

\section{IMAGES IN CARDIOLOGY}

\section{Probable intracardiac tuberculoma in an HIV positive woman}

A 50 year old Zambian woman presented in August 1997 with fever and breathlessness. Echocardiography showed a left pleural effusion and a small pericardial effusion. Pleural fluid was exudative and lymphocytic, highly suggestive of tuberculosis. Culture for acid fast bacilli was negative. A clinical diagnosis of tuberculosis was made and she was prescribed quadruple antituberculous treatment. She was



seropositive for the human immunodeficiency virus (HIV) with a reduced CD4 lymphocyte count of 220 cells/ $\mu$ l.

There was a good clinical response to antituberculous treatment with resolution of fever and pleural effusion. Combination antiretroviral treatment was started in November 1997. Repeat echocardiography in December 1997 (below left) revealed a new $2 \times 3 \mathrm{~cm}$ mass in the wall of the right atrium involving the base of the tricuspid valve. The position of the mass within the right atrial wall was confirmed by cardiac magnetic resonance imaging. The patient declined invasive tests and was managed conservatively. There was no clinical, haematological, or microbiological evidence to suggest additional opportunistic infections, Kaposi's sarcoma, or lymphoma.

Repeat echocardiography in April 1998 (below right) revealed spontaneous resolution of the mass. We have been unable to find any similar cases in the literature in either HIV positive or negative individuals. Presentation with new intracranial tuberculomas during treatment followed by resolution is well documented. Although there is no biopsy of the intracardiac lesion the clinical response to empirical antituberculous treatment in the absence of other disease strongly suggests that this lesion was a cardiac tuberculoma.
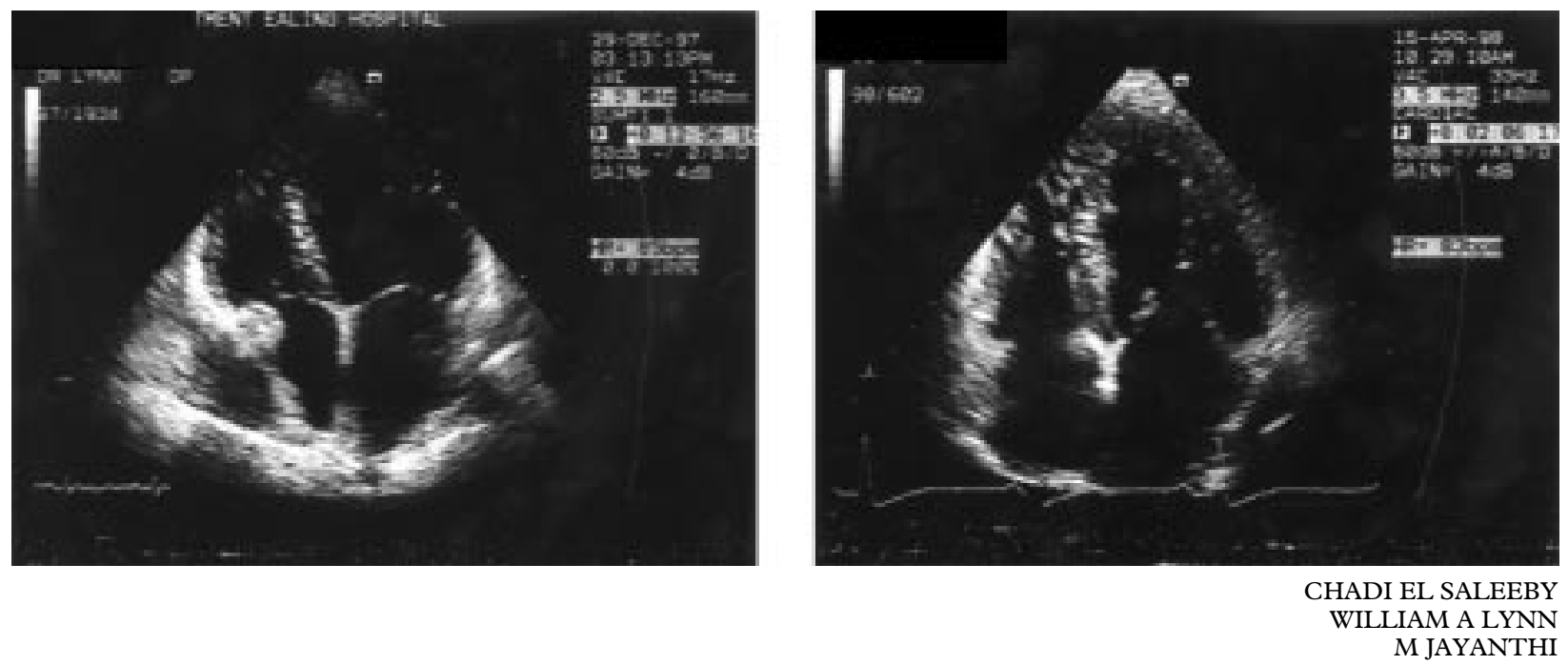